\title{
Palliative Care and Life-Sustaining/Local Procedures in Colorectal Cancer in the United States Hospitals: A Ten-Year Perspective
}

\author{
Zahra Mojtahedi (D) \\ Ja Seol Koo (D) ${ }^{1,2}$ \\ Ji Yoo (D) ${ }^{3}$ \\ Pearl Kim' \\ Hee-Taik Kang (D) ${ }^{4}$ \\ Jinwook Hwang ${ }^{5}$ \\ Moon Kyung Joo 6 \\ Jay J Shen (D)
}

'Department of Healthcare

Administration and Policy, School of

Public Health, University of Nevada, Las

Vegas, NV, 89119, USA; ${ }^{2}$ Division of

Gastroenterology and Hepatology,

Department of Internal Medicine, Korea

University Ansan Hospital, Ansan, South

Korea; ${ }^{3}$ Department of Internal Medicine,

University of Nevada Las Vegas School of

Medicine, Las Vegas, NV, USA;

${ }^{4}$ Department of Family Medicine,

Chungbuk National University Hospital,

Cheongju, South Korea; ${ }^{5}$ Department of

Cardiovascular and Thoracic Surgery,

Korea University Ansan Hospital, Ansan,

South Korea; ${ }^{6}$ Division of

Gastroenterology, Department of Internal Medicine, Korea University

College of Medicine, Seoul, South Korea

Correspondence: Ja Seol Koo

Division of Gastroenterology and

Hepatology, Department of Interna

Medicine, Korea University Ansan

Hospital, Ansan, Gyeonggi-do, I5355,

South Korea

Email jskoo@korea.ac.kr

Jay J Shen

Department of Healthcare Administration and Policy, School of Public Health,

University of Nevada, Las Vegas, NV,

89119, USA

Email jay.shen@unlv.edu
Background: In recent years, palliative care utilization has been increasing while lifesustaining/local procedures have been declining at the end of life. Palliative care utilization widely varies based on tumor type. Limited information is available on inpatient palliative care in colorectal cancer.

Aims: This study investigated inpatient palliative care utilization and its association with patient demographics, hospital charges, and procedures among colorectal cancer patients admitted to US hospitals between 2008 and 2017. Receipt of life-sustaining and local procedures and surgeries were also investigated during the ten years.

Methods: Data were extracted from the National inpatient sample (NIS) database containing de-identified information from each hospitalization. Codes V66.7 for ICD-9-CM or Z51.5 for ICD-10-CM were used to find palliative care utilization. Data were analyzed using generalized regression with adjustment for variations in predictors. The Compound Annual Growth Rate (CAGR) was calculated for palliative care and procedures over time.

Results: Of the 487,027 colorectal cancer hospitalizations, only $6.04 \%$ utilized palliative care. This percentage significantly increased over time from $2.3 \%$ in 2008 to $9.3 \%$ in 2017 $(P<0.0001)$. Palliative care utilization sizably decreased hospital charges by $\$ 18,010$ per hospitalization $(P<0.0001)$ and was positively associated with female gender, severe disease, and age over 80 years $(P \leq 0.05)$. Palliative care utilization was inversely associated with using life-sustaining and local procedures and surgeries $(P<0.0001)$. Life-sustaining procedures (intubation, infusion of concentrate nutrients, dialysis, and blood transfusion) and surgeries were decreased over time $(P<0.001)$.

Conclusions: Palliative care utilization increased over time and was inversely associated with hospital charges and performing procedures among colorectal cancer patients. Our findings warrant further research and interventions to increase palliative care utilization in colorectal cancer.

Keywords: colorectal cancer, financial burden, hospital charges, palliative care, procedures, public health

\section{Introduction}

Palliative care provides comfort and improves the quality of life for patients dealing with terminal illnesses, ideally throughout the course of the disease. The palliative care team consists of physicians, nurses, social workers, and other specialties working together to alleviate pain as well as psychological and spiritual distress of patients with terminal illnesses. ${ }^{1-5}$ This symptom management has been associated with a reduction in non-beneficial procedures and also hospital charges and 
length of stay (LOS). ${ }^{1-5}$ The utilization of palliative care mainly varies by gender, race/ethnicity, educational level, obesity, socioeconomic status, health insurance type, and discussion by physicians in the last year of life. ${ }^{6-11}$ Additionally, palliative care usage varies widely based on tumor type, ${ }^{12}$ indicating that each cancer needs to be investigated in order to identify appropriate interventions for promoting palliative care where it is underutilized.

Colon cancer has been on the rise in recent decades across the world. In the USA, it is the third most common cancer in both men and women. Generally, if colon cancer is diagnosed early, the tumor can be removed by surgery, with a 5-year survival of up to $60 \% .{ }^{13}$ However, end-stage colorectal cancer patients suffer from obstruction and colostomy in addition to other distressful symptoms. Fear of leakage, embarrassment caused by noises, gas, and odor, need for increased privacy can lead to social isolation, depression, and anxiety. ${ }^{14,15}$ These patients can thus be appropriate candidates for palliative care to improve their quality of life.

A nationally representative database in the USA is the national inpatient sample (NIS). ${ }^{6-8}$ The NIS dataset also facilitates reliable comparisons across studies. Using the NIS dataset, it has been revealed that palliative care utilization has increased in recent decades in the USA, but varies widely based on tumor type, with documented utilization ranging from $4.9 \%$ in breast cancer hospitalizations to $16 \%$ in liver cancer hospitalizations. ${ }^{12}$ The underlying reasons for such variations have not been fully explained, but the variations may be related to differences in prognosis, distressful symptoms, or patient demographics. Previous research found a 5\% utilization of inpatient palliative care for colorectal cancer patients between 2004 and 2016. ${ }^{12}$ However, data are not available in detail to provide insight about demographics, dying status, gender, etc. of the patients.

In the current study, using V66.7 (ICD-9, before Oct 2015) and Z51.5 (ICD-10, after Oct 2015) codes within the NIS database, we aimed to characterize the extent of utilization of inpatient palliative care services among colorectal cancer patients and its association with gender, age, race/ethnicity, hospital charges, payer source, the severity of disease, life-sustaining, and local procedures, and surgery. Temporal trends of palliative care and procedures were also determined for the study period.

\section{Methods}

\section{Study Design}

A pooled, retrospective, cross-sectional study was conducted based on hospital discharge data retrieved from the NIS, a database that is part of the Healthcare charge and Utilization Project (HCUP). The NIS is a secondary dataset and contains data from over seven million hospital stays each year in the USA. It represents a $20 \%$ sample from hospital stays.

Our research was conducted in accordance with the Declaration of Helsinki. Upon completion of a data user agreement with the Agency for Healthcare Research and Quality, the sponsoring agency for HCUP, completely deidentified data was delivered. Therefore, the NIS data are interpretable for each hospitalization, and possible readmitted cases are considered new hospitalization. The Institutional Review Board at the University of Nevada, Las Vegas found the current study to be exempt with negligible risks to subjects.

\section{Study Population and Variables}

The study period was from 2008 to 2017. International Classification of Diseases, 9th revision, Clinical Modification (ICD-9-CM) and ICD-10.CM codes were used to identify colorectal cancer (Supplementary File 1). Hospitalizations with ICD-9 or ICD-10 codes for colorectal cancer were included. Patients younger than 18 years or missing demographic data were excluded. Extracted variables of interest for each hospitalization included age group $(<30$ years old, 40-49, 50-59, 60-69, 70-79, and $\geq 80$ years old), gender, race, quartile of median income by zip codes, the severity of illness (All Patient Refined Diagnosis-Related Group [APR-DRG]), primary payer (Medicare, Medicaid, private insurance, other), the number of diagnoses/comorbidities, metastasis, LOS, in-hospital death, hospital size, hospital locations and regions, hospital charges, local procedure (endoscopy, stent insertion, and drainage), life-sustaining procedures (intubation, infusion, infusion of concentrate nutrients, dialysis, blood transfusion, and cardiopulmonary resuscitation [CPR]), and surgeries (resection, bypass, and colostomy creation). APR-DRG has four levels of 1 to 4, indicating minor, moderate, major, and extreme loss of function. ICD9-CM and ICD-10.CM codes used to extract palliative care and procedures are shown in Supplementary File 1. Patients, at discharge, are coded for palliative care in the NIS database when terms such as palliative care, comfort 
care, end-of-life care, hospice, or similar terms are written in their records. ${ }^{6-8}$ Therefore, these codes cover a range of palliative care services from consultation to full services. Total hospital charges were adjusted for the annual hospital expenditure growth rate provided by the Center for Medicare and Medicaid Services each year. ${ }^{6,16,17}$

\section{Statistical Analyses}

Generalized regression analysis with patient characteristics as the individual level and hospital characteristics as the hospital level was conducted, with adjustment for variations in predictors. The hospital was the random effect in the generalized modeling to control for the potential within-hospital clustering effects; with other variables being included as the fixed effects. The link function was Logit. The main outcomes were receipt of palliative care, palliative procedures, and hospital charges, and death during hospitalization. All covariates were categorical except for three ordinal variables, age group (1-7), APR-DRG Severity score (1-4), and quartiles of median income by zip code (1-4). Odds ratios (ORs) and their corresponding 95\% confidence intervals (CIs) were computed for predictors. The compound annual growth rate (CAGR) was calculated using Excel software to calculate temporal trends. The formula for CAGR is $(\mathrm{y} / \mathrm{x}),{ }^{1 /(\mathrm{B}-\mathrm{A})-1}$ where year $A$ is $x$ and year $B$ is $y$. The statistical significance of CAGR was tested using Rao-Scott correction for $\chi^{2}$ tests for categorical variables. ${ }^{6}$ All reported $P$-values were 2 -tailed; $P$-values $<0.05$ were considered statistically significant.

\section{Results}

Totally, 487, 027 colorectal cancer hospitalizations with complete data constitute our study group. Their characteristics are demonstrated in Table 1. The mean age was $66.5 \pm$ 13.9 years, and men comprised $52 \%$ of our study population. As the median household income increased, the colorectal cancer rate also increased (21.7\% in the lowest percentile of income and $28.2 \%$ in the highest percentile of income). Inpatient palliative care was utilized by $6.7 \%$ of patients (Table 1). The majority of patients were white $(71.7 \%)$, had Medicare (55.5\%), were admitted to large hospitals (59.6\%) located in rural areas (55.8\%) and the South (38.9\%).

The CAGRs of intubation, infusion, infusion of concentrate nutrients, dialysis, blood transfusion, and CPR were $-2.30 \%, 12.27 \%-5.21 \%,-11.53 \%,-5.28 \%$, and $-0.76 \%$, respectively. Intubation, infusion, infusion of concentrate nutrients, dialysis, and blood transfusion significantly changed over time $(P<0.001)$, but $C P R$ remained unchanged $(P=0.198)$. Figure 1 presents the CAGRs of pooled life-sustaining procedures, local procedures, surgeries, and palliative care with CAGRs of $-3.9 \%$ $(P<0.001), 0.31 \% \quad(P=0.015), \quad-0.62 \% \quad(P<0.001)$, and $14.9 \% \quad(P<0.001)$, respectively. All were significantly changed over time $(P<0.05)$.

Palliative care utilization significantly increased over time $(\mathrm{OR}=1.09, \mathrm{CI}=1.08-1.10, P<0.0001)$. As the age increased, the odd of receiving palliative care increased by $22 \%(\mathrm{OR}=1.22, \mathrm{CI}=1.21-1.24, P<0.0001)$. Other Factors associated with a higher receipt of palliative care included female gender $(\mathrm{OR}=1.17, \mathrm{CI}=1.14-1.20, P<0.0001)$, black race compared to whites $(\mathrm{OR}=1.10, \mathrm{CI}=1.06-1.15$, $P<0.0001)$, Hispanics compared to whites $(\mathrm{OR}=1.05$, $\mathrm{CI}=$ 1.00-1.11, $P=0.0350$ ), Asians/ Pacific Islanders (PI) compared to whites $(\mathrm{OR}=1.18, \mathrm{CI}=1.10-1.27, P<0.0001)$, disease severity $(\mathrm{OR}=1.80$ as the severity increased one level higher, $\mathrm{CI}=1.77-1.84, \quad P<0.0001)$, metastasis $(\mathrm{OR}=2.31, \mathrm{CI}=2.25-2.37, P<0.0001)$, and uninsured patients $(\mathrm{OR}=1.31$ as compared to patients with private insurance, $\mathrm{CI}=1.21-1.42, P<0.0001)$. Medicare beneficiaries significantly used less palliative care services compared to patients with private insurance $(\mathrm{OR}=0.67, \mathrm{CI}=$ 0.65-0.69, $P<0.0001)$.

Life-sustaining procedures, local procedures, surgeries, small and medium hospitals compared to large hospitals, and rural and urban non-teaching hospitals compared to urban-teaching hospitals all were significantly associated with lower palliative care utilization $(P<0.0001)$. The quartile of median income was not associated with palliative care utilization $(P=0.2851)$ (Table 2$)$.

As it is indicated in Table 3, local procedures significantly decreased over time and their utilization was significantly lower in women than in men and blacks and Hispanics than in whites $(P<0.05)$. Asians/ PI significantly utilized more local procedures than whites $(P<$ 0.0001). Patients with Medicare, Medicaid, no insurance, and no charges, compared to private insurance, and metastasis significantly had lower utilization of local procedures $(P<0.0001)$. The severity of illness, number of diagnoses/ comorbidities, and higher quartile of median income were significantly associated with higher utilization of local procedures $(P<0.0001)$. Small and medium hospitals compared to large hospitals, rural and urban nonteaching hospitals compared to urban-teaching hospitals, and hospitals in Midwest compared to the South had significantly lower utilization of local procedures $(P<0.05)$. 
Table I Characteristics of Colorectal Cancer Patients (The NIS Dataset; Weighted Number, 2,478,432)

\begin{tabular}{|c|c|c|c|c|c|}
\hline Characteristics & 2008-2017 & 2008 & 2011 & 2014 & 2017 \\
\hline \multicolumn{6}{|l|}{ Gender \% (n) } \\
\hline Male & $52.0(1,290,235)$ & $51.2(133,291)$ & $5 I .3(129,57 \mid)$ & $52.0(122,475)$ & $52.8(127,785)$ \\
\hline Female & $48.0(I, \mid 86,526)$ & $48.7(126,974)$ & $48.6(122,719)$ & $47.9(112,880)$ & $47.1(114,035)$ \\
\hline Age, mean (SD) & $66.5(31.1)$ & $67.6(31.3)$ & $67.1(30.8)$ & $66.2(31.0)$ & $65.8(31.0)$ \\
\hline \multicolumn{6}{|l|}{ Age groups \% (n) } \\
\hline$<30$ & $0.6(16,292)$ & $0.6(1667)$ & $0.6(1583)$ & $0.6(1519)$ & $0.7(1614)$ \\
\hline $30-39$ & $2.5(63,278)$ & $2.3(6001)$ & $2.3(5835)$ & $2.6(6194)$ & $2.7(6740)$ \\
\hline $40-49$ & $8.4(208,178)$ & $8.0(21,012)$ & $8.0(20,403)$ & $8.3(19,680)$ & $8.4(20,409)$ \\
\hline $50-59$ & $19.5(484,084)$ & $17.9(46,824)$ & I9.I (48,440) & $20.1(47,435)$ & $19.8(48,054)$ \\
\hline $60-69$ & $25.0(621,487)$ & $23.1(60,377)$ & $24.2(6 I, 34 I)$ & $25.5(60,110)$ & $26.5(64,284)$ \\
\hline $70-79$ & $23.6(586,033)$ & $24.8(200,523)$ & $23.9(60,4 \mid 2)$ & $23.3(54,970)$ & $23.6(57,129)$ \\
\hline$\geq 80$ & $20.1(499,080)$ & $23.0(260,504)$ & $21.5(54,354)$ & $19.3(45,465)$ & $18.0(43,619)$ \\
\hline \multicolumn{6}{|l|}{ Race \% (n) } \\
\hline White & $71.7(1,630,625)$ & $74.0(153,957)$ & $72.3(16,747)$ & $72.5(16,215)$ & $70.1(164,364)$ \\
\hline Black & I3.4 (304,890) & $12.0(25,009)$ & $14.2(32,978)$ & $12.9(28,855)$ & I3.I (30,930) \\
\hline Hispanic & $8.3(188,970)$ & $7.2(15,014)$ & $7.9(18,327)$ & $8.4(18,835)$ & $9.3(21,860)$ \\
\hline Asian/Pacific Islander & $3.1(72,487)$ & 3.1 (6549) & $2.6(6232)$ & $3.2(7 \mid 95)$ & $3.6(8569)$ \\
\hline Native Americans/others & $0.5(11,342)$ & $0.4(904)$ & $0.4(875)$ & $0.4(939)$ & $0.5(1150)$ \\
\hline \multicolumn{6}{|l|}{ Payer source \% (n) } \\
\hline Medicare & $55.5(1,373,744)$ & $56.8(148,053)$ & $56.9(14,343)$ & $55.2(129,660)$ & $55.1(133,085)$ \\
\hline Medicaid & $9.4(234,4 \mid 2)$ & $7.3(18,989)$ & $8.3(2 I, 07 I)$ & $10.4(24,420)$ & $10.9(26,364)$ \\
\hline Private insurance & $29.4(728,593)$ & $30.4(79,211)$ & $29.0(73,105)$ & $29.5(69,260)$ & $29.3(70,914)$ \\
\hline Uninsured & $2.7(68,872)$ & $2.1(5510)$ & $2.7(6956)$ & $2.3(5540)$ & $2.2(5395)$ \\
\hline No charge & $0.3(8529)$ & $0.4(1015)$ & $0.5(1230)$ & $0.3(724)$ & $0.2(500)$ \\
\hline Other & $2.4(60,036)$ & $2.8(7459)$ & $2.4(6138)$ & $2.2(5264)$ & $2.1(5209)$ \\
\hline \multicolumn{6}{|l|}{ Median household income by zip code \% (n) } \\
\hline 76th to 100th percentile & $28.2(684,373)$ & $26.5(68,978)$ & $28.4(71,658)$ & $27.6(64,965)$ & $28.6(69,155)$ \\
\hline 5 Ist to 75 th percentile & $26.1(633,419)$ & $27.8(72,579)$ & $24.5(6 I, 93 I)$ & $27.8(65,350)$ & $26.2(63,529)$ \\
\hline 26th to 50th percentile & $23.9(581,025)$ & $23.2(60,489)$ & $25.7(65,029)$ & $23.3(43,790)$ & $23.9(57,894)$ \\
\hline 0th to 25 th percentile & $21.7(526,711)$ & $22.4(58,447)$ & $21.3(53,907)$ & $21.3(50.160)$ & $21.2(51,234)$ \\
\hline \multicolumn{6}{|l|}{ Severity of illness \% (n) } \\
\hline APR-DRG I & $14.1(35|| 22)$, & $14.9(38,967)$ & $13.7(34,683)$ & $13.6(32,150)$ & I2.4 (30,000) \\
\hline APR-DRG 2 & $39.9(990,107)$ & $42.0(109,522)$ & $38.1(96,231)$ & $39.5(93,025)$ & $37.6(91,064)$ \\
\hline APR-DRG 3 & $34.5(856,072)$ & $32.6(85,06 I)$ & $35.4(89,386)$ & $35.8(840.240)$ & $36.9(89,189)$ \\
\hline APR-DRG 4 & $11.3(28 I, 129)$ & $10.3(26,953)$ & $12.7(32,249)$ & II.0 $(25,960)$ & $13.0(31,564)$ \\
\hline Metastasis \% (n) & $29.9(742,485)$ & $27.6(71,944)$ & $28.7(72,463)$ & $30.2(7|, 2| 0)$ & $32.2(78,024)$ \\
\hline Palliative care consultation \% (n) & $6.0(149,707)$ & $2.3(5998)$ & $5.3(13,374)$ & $7.4(17,630)$ & $9.2(22,440)$ \\
\hline Life-sustaining procedures \% (n) & $24.1(598,5 \mid 2)$ & $26.4(68,748)$ & $27.6(69,876)$ & $24.0(56,390)$ & $17.0(41,119)$ \\
\hline Local procedures \% (n) & $10.1(251,653)$ & $19.0(26,055)$ & $10.6(26,758)$ & $10.9(25,755)$ & $10.6(25,729)$ \\
\hline Surgeries \% (n) & $36.6(909,254)$ & $45.6(118,940)$ & $45.4(1|4,8| 3)$ & $44.4(19,46 \mid)$ & $36.2(87,644)$ \\
\hline Number of diagnoses/comorbidities, mean (SD) & $11.7(13.8)$ & $9.6(11.2)$ & $11.1(12.8)$ & $12.6(14.2)$ & $13.9(15.4)$ \\
\hline LOS, mean (std.) & $7.0(16.0)$ & $7.7(16.9)$ & $7.2(17.0)$ & $6.9(15.2)$ & $6.5(15.0)$ \\
\hline Total charges, mean \$ (SD) & $76,505(218,107)$ & $78,893(223,242)$ & $77,353(225,263)$ & $76,075(211,823)$ & $75,599(215,997)$ \\
\hline
\end{tabular}

(Continued) 
Table I (Continued).

\begin{tabular}{|c|c|c|c|c|c|}
\hline Characteristics & 2008-2017 & 2008 & 2011 & 2014 & 2017 \\
\hline In-hospital death \% (n) & $4.4(110,763)$ & $5.0(13,080)$ & $4.5(11,460)$ & $4.2(9900)$ & $4.2(10,164)$ \\
\hline \multicolumn{6}{|l|}{ Hospital bed size \% (n) } \\
\hline Small & $14.4(355,069)$ & II.8 (30.904) & II $.4(28,447)$ & I7.7 $(4 \mid, 740)$ & $17.5(42,443)$ \\
\hline Medium & $25.9(640,95 I)$ & $23.1(60,299)$ & $24.3(60,594)$ & $28.0(65,950)$ & $28.6(69,159)$ \\
\hline Large & $59.6(1,472,027)$ & $64.9(168,939)$ & $64.2(160,202)$ & $54.2(127,585)$ & $53.8(130,215)$ \\
\hline \multicolumn{6}{|c|}{ Hospital location/teaching status \% (n) } \\
\hline Urban non-teaching & $10.9(270,823)$ & $12.7(33,109)$ & I I.4 $(28,495)$ & $9.7(22,894)$ & $8.6(20,849)$ \\
\hline Urban teaching & $33.1(818,200)$ & $42.4(110,414)$ & $40.4(100,834)$ & $24.8(58,529)$ & $20.9(50,760)$ \\
\hline Rural & $55.8(1379,023)$ & $44.8(116,619)$ & $48.1(119,9 \mid 4)$ & $65.4(153,950)$ & $70.4(170,209)$ \\
\hline \multicolumn{6}{|l|}{ Hospital region } \\
\hline Northeast & $19.7(488,873)$ & $18.9(49,352)$ & $19.8(20,125)$ & $19.3(45,550)$ & $19.0(46,164)$ \\
\hline Midwest & $23.1(574,131)$ & $23.9(62,414)$ & $22.8(57,7 \mid 3)$ & $22.8(53,859)$ & $22.5(54,499)$ \\
\hline South & $38.9(964,613)$ & $39.2(102,128)$ & $39.9(100,885)$ & $39.3(92,476)$ & $39.0(94,440)$ \\
\hline West & I8.| $(450,8 \mid 3)$ & $17.8(46,609)$ & I7.3 $(43,826)$ & I8.4 (43,489) & $19.3(46,7 \mid 4)$ \\
\hline
\end{tabular}

Abbreviations: NIS, National Inpatient Sample; SD, standard deviation; APR-DRG, all-patient refined diagnosis-related group.

Table 4 displays factors associated with hospital charges among colorectal cancer patients. Palliative care was associated with a reduction of $\$ 18,010$ per hospitalization $(P<0.0001)$. A significant decrease in hospital charges over time for colorectal cancer patients was observed after adjustment for the health inflation rate $(P<0.0001)$. Being a woman compared to men was associated with reduced hospital charges $(P<0.0001)$. Medicare and Medicaid beneficiaries and uninsured patients had significantly lower hospital charges compared to private insurance $(P<0.0001)$. Lower hospital charges were significantly reported in small and medium hospitals (as compared to large hospitals), rural and urban nonteaching hospitals (as compared to urban-teaching hospitals), and Midwest hospitals (as compared to South hospitals) ( $P<$ 0.0001). Blacks, Hispanics, and Asians/PI Islanders had significantly higher hospital charges compared to whites $(P<$ $0.0001)$. As the severity of the illness or the number of diagnoses/comorbidities increased, hospital charges significantly increased $(P<0.001)$. In-hospital death, life-sustaining procedures, local procedures, surgeries, higher quartiles of

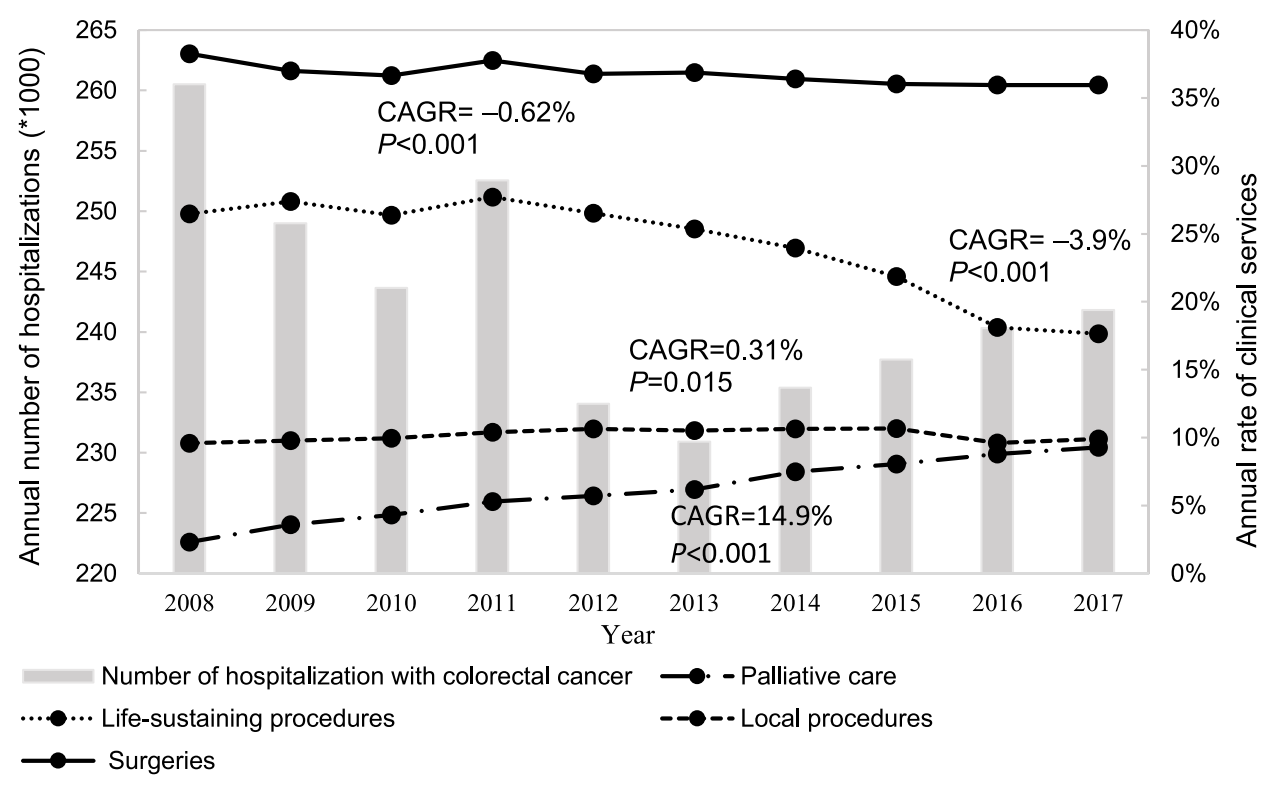

Figure I Compound annual growth rates of pooled life-sustaining and local procedures, surgeries, and palliative care in colorectal cancer patients. 
Table 2 Factors Associated with Inpatient Palliative Care Consultation in Colorectal Cancer $(n=487,027)$

\begin{tabular}{|c|c|c|c|}
\hline Independent Variable & OR & $95 \% \mathrm{Cl}$ & $P$-value \\
\hline Year & 1.09 & $1.08-1.10$ & $<0.0001$ \\
\hline Age group & 1.22 & $1.16-1.24$ & $<0.0001$ \\
\hline Female & 1.17 & $1.14-1.20$ & $<0.0001$ \\
\hline \multicolumn{4}{|l|}{ Race } \\
\hline White (reference) & 1.00 & & \\
\hline Black & 1.10 & $1.06-1.15$ & $<0.0001$ \\
\hline Hispanic & 1.05 & $1.00-1.11$ & 0.035 \\
\hline Asian/Pacific Islander & 1.18 & $1.10-1.27$ & $<0.0001$ \\
\hline Other & 0.96 & $0.89-1.04$ & 0.4166 \\
\hline \multicolumn{4}{|l|}{ Primary payer } \\
\hline Private insurance (reference) & 1.00 & & \\
\hline Medicare & 0.67 & $0.65-0.69$ & $<0.0001$ \\
\hline Medicaid & 1.03 & $0.98-1.108$ & 0.1698 \\
\hline Uninsured & 1.31 & $1.21-1.42$ & $<0.0001$ \\
\hline No charge & 0.71 & $0.53-0.95$ & 0.0236 \\
\hline Other & 2.58 & $2.4 I-2.76$ & $<0.0001$ \\
\hline Severity of illness: APR-DRG & 1.80 & $1.77-184$ & $<0.0001$ \\
\hline Metastasis & 2.31 & $2.25-32.37$ & $<0.0001$ \\
\hline Number of diagnoses/comorbidities & 1.06 & $1.06-1.07$ & $<0.0001$ \\
\hline Life-sustaining procedures & 0.89 & $0.86-0.92$ & $<0.0001$ \\
\hline Local procedures & 0.84 & $0.80-0.87$ & $<0.0001$ \\
\hline Surgeries & 0.23 & $0.22-0.24$ & $<0.0001$ \\
\hline Quartile of median income by zip code & 1.00 & $0.99-1.02$ & 0.2851 \\
\hline \multicolumn{4}{|l|}{ Hospital bed size } \\
\hline Large (reference) & 1.00 & & \\
\hline Small & 0.85 & $0.82-0.89$ & $<0.0001$ \\
\hline Medium & 0.94 & $0.89-0.96$ & 0.0029 \\
\hline \multicolumn{4}{|l|}{ Hospital location/teaching status } \\
\hline Urban teaching (reference) & 1.00 & & \\
\hline Rural & 0.79 & $0.75-0.84$ & $<0.0001$ \\
\hline Urban nonteaching & 0.85 & $0.82-0.88$ & $<0.0001$ \\
\hline \multicolumn{4}{|l|}{ Hospital region } \\
\hline South (reference) & 1.00 & & \\
\hline Northeast & 1.07 & $1.00-1.13$ & 0.0264 \\
\hline Midwest & 0.95 & $0.90-1.01$ & 0.1325 \\
\hline West & 1.13 & $1.07-1.20$ & $<0.0001$ \\
\hline
\end{tabular}

Abbreviations: OR, odds ratio; $\mathrm{Cl}$, confidence interval; APR-DRG, all patient refined-diagnosis-related group.

median income were significantly associated with higher hospital charges $(P<0.0001)$.

\section{Discussion}

We investigated temporal trends of palliative care utilization among colorectal cancer patients from 2007 to 2018. We found that its usage increased over time, but utilization
Table 3 Factors Associated with Local Procedures in Colorectal Cancer $(n=487,027)$

\begin{tabular}{|c|c|c|c|}
\hline Independent Variables & OR & $95 \% \mathrm{Cl}$ & P-value \\
\hline Year & 0.94 & $0.93-0.94$ & $<0.0001$ \\
\hline Age group & 1.02 & $1.01-1.03$ & $<0.0001$ \\
\hline Female & 0.98 & $0.97-0.99$ & 0.020 \\
\hline \multicolumn{4}{|l|}{ Race } \\
\hline White (reference) & 1.00 & & \\
\hline Black & 0.95 & $0.93-0.0 .97$ & $<0.0001$ \\
\hline Hispanic & 0.90 & $0.88-0.0 .92$ & $<0.0001$ \\
\hline Asian/Pacific Islander & 1.10 & $1.06-1.14$ & $<0.0001$ \\
\hline Other & 0.99 & $0.96-0.1 .00$ & 0.8700 \\
\hline \multicolumn{4}{|l|}{ Primary payer } \\
\hline Private insurance (reference) & 1.00 & & \\
\hline Medicare & 0.84 & $0.83-0.86$ & $<0.0001$ \\
\hline Medicaid & 0.66 & $0.64-0.67$ & $<0.0001$ \\
\hline Uninsured & 0.82 & $0.79-0.85$ & $<0.0001$ \\
\hline No charge & 0.70 & $0.63-0.77$ & $<0.0001$ \\
\hline Other & 0.65 & $0.62-0.68$ & $<0.0001$ \\
\hline Severity of illness: APR-DRG & 1.08 & $1.07-1.10$ & $<0.0001$ \\
\hline Metastasis & 0.34 & $0.33-0.34$ & $<0.0001$ \\
\hline Number of diagnoses/comorbidities & 1.00 & $1.00-101$ & $<0.0001$ \\
\hline In-hospital death & 1.11 & $1.08-1.14$ & $<0.0001$ \\
\hline Quartile of median income by zip code & 1.02 & $1.01-1.03$ & $<0.0001$ \\
\hline \multicolumn{4}{|l|}{ Hospital bed size } \\
\hline Large (reference) & 1.00 & & \\
\hline Small & 0.83 & $0.81-0.85$ & $<0.0001$ \\
\hline Medium & 0.97 & $0.950-0.0 .99$ & 0.0008 \\
\hline \multicolumn{4}{|l|}{ Hospital location/teaching status } \\
\hline Urban teaching (reference) & 1.00 & & \\
\hline Rural & 0.74 & $0.73-0.76$ & $<0.0001$ \\
\hline Urban nonteaching & 0.94 & $0.92-0.96$ & $<0.0001$ \\
\hline \multicolumn{4}{|l|}{ Hospital region } \\
\hline South (reference) & 1.00 & & \\
\hline Northeast & 0.98 & $0.95-0.100$ & 0.1359 \\
\hline Midwest & 0.93 & $0.90-0.95$ & $<0.0001$ \\
\hline West & 1.09 & $1.05-1.12$ & $<0.0001$ \\
\hline
\end{tabular}

Abbreviations: OR, odds ratio; $\mathrm{Cl}$, confidence interval; APR-DRG, all patient refined-diagnosis-related group.

of life-sustaining and surgeries were decreased. Palliative care was also positively associated with reduced hospital charges, female gender, older ages, the severity of illness, metastasis, and the number of diagnoses/comorbidities. Palliative care was inversely associated with lifesustaining and local procedures, surgeries, hospitalization at small/medium hospitals compared to large hospitals, and at rural/non-teaching urban hospitals compared to urban-teaching hospitals. 
Table 4 Factors Associated with Hospital Charges in Colorectal Cancer

\begin{tabular}{|c|c|c|c|}
\hline Independent Variable & $\begin{array}{c}\text { Coefficient, } \\
\beta\end{array}$ & $\begin{array}{c}\text { Standard } \\
\text { Error }\end{array}$ & $P$-value \\
\hline Year & -1801 & 48 & $<0.000$ \\
\hline Age group & -3076 & 128 & $<0.000$ \\
\hline Female & -3883 & 262 & $<0.000$ \\
\hline \multicolumn{4}{|l|}{ Race } \\
\hline White (reference) & 1.00 & & \\
\hline Black & 2273 & 422 & $<0.000$ \\
\hline Hispanic & 12,868 & 521 & $<0.000$ \\
\hline Asian/Pacific Islander & 12,247 & 815 & $<0.000$ \\
\hline Other & 7888 & 780 & $<0.000$ \\
\hline \multicolumn{4}{|l|}{ Primary payer } \\
\hline Private insurance (reference) & 1.00 & & \\
\hline Medicare & -4485 & 370 & $<0.000$ \\
\hline Medicaid & -5602 & 501 & $<0.000$ \\
\hline Uninsured & -4657 & 826 & $<0.000$ \\
\hline No charge & -5993 & 2230 & 0.0072 \\
\hline Other & $-10,523$ & 873 & $<0.000$ \\
\hline Severity of illness: APR-DRG & 27,204 & 210 & $<0.000$ \\
\hline Metastasis & $-29,263$ & 298 & $<0.000$ \\
\hline Number of diagnoses/comorbidities & 2263 & 30. & $<0.000$ \\
\hline In-hospital death & 8766 & 654 & $<0.000$ \\
\hline Palliative care & $-18,010$ & 560 & $<0.000$ \\
\hline Life-sustaining procedures & 32,505 & 316 & $<0.000$ \\
\hline Local procedures & 22,298 & 411 & $<0.000$ \\
\hline Surgeries & 50,683 & 271 & $<0.000$ \\
\hline $\begin{array}{l}\text { Quartile of median income by zip } \\
\text { code }\end{array}$ & 649 & 128 & $<0.000$ \\
\hline \multicolumn{4}{|l|}{ Hospital bed size } \\
\hline Large (reference) & 1.00 & & \\
\hline Small & $-10,306$ & 386 & $<0.000$ \\
\hline Medium & -7117 & 310 & $<0.000$ \\
\hline \multicolumn{4}{|l|}{ Hospital location/teaching status } \\
\hline Urban teaching (reference) & 1.00 & & \\
\hline Rural & $-28,050$ & 453 & $<0.000$ \\
\hline Urban nonteaching & -3792 & 297 & $<0.000$ \\
\hline \multicolumn{4}{|l|}{ Hospital region } \\
\hline South (reference) & 1.00 & & \\
\hline Northeast & 10,388 & 375 & $<0.000$ \\
\hline Midwest & -8653 & 355 & $<0.000$ \\
\hline West & 28,907 & 398 & $<0.000$ \\
\hline
\end{tabular}

Abbreviation: APR-DRG, All patient refined-diagnosis-related group.

Rubens et al investigated palliative care utilization in overall common cancers, using the same dataset as ours, between 2005 and $2014 .{ }^{12}$ They found the utilization of palliative care in colorectal cancer was $5 \%$, which was lower than the national average of $9.9 \%$ in cancer. ${ }^{12}$ Our finding on colorectal cancer is $1.7 \%$ higher than theirs that might be explained by more updated findings of the current study since palliative care utilization has been increasing in recent years. ${ }^{6-8,12,18-20}$ Health insurance policies are increasingly promoting payments based on diagnosis rather than traditional fee-for-services in the USA. ${ }^{16}$ This might be an underlying reason for increasing palliative care and decreasing non-beneficial life-sustaining procedures at the end of life, a finding that has been frequently reported in a wide range of disorders from cancers to noncancer conditions in recent years. ${ }^{6-8,12,17,18}$ Improving acceptance of the importance of palliative care services and access to these services are other reasons for the upward trend of palliative care utilization in recent years. ${ }^{8}$ We found that the utilization of life-sustaining procedures (intubation, infusion of concentrate nutrients, dialysis, and blood transfusion) was reduced in the patients over time. Interestingly, performing life-sustaining or local procedures was negatively associated with palliative care utilization, implying that these procedures might have been conducted as an alternative to palliative care. However, the palliative care team provides emotional support and pain management and also determines the goal of care, ${ }^{1-5}$ the services that are not delivered through other procedures. Therefore, palliative care should be encouraged even among patients undergoing life-sustaining and local procedures. Surgery was conducted in almost $37 \%$ of colorectal cancer patients during their admission and was inversely and strongly associated with a reduction in palliative care utilization, implying that surgery was likely curative, at least in some patients, with no need for palliative care during that hospital stay.

The receipt of palliative care in colorectal cancer in our study was very low compared to other cancers and was close to previous reports on colorectal cancer, ${ }^{12}$ which might be related to the overall good prognosis of the disease. However, almost, $30 \%$ of our patients were metastatic and $46 \%$ had a moderate to severe loss of function (APRDG 3 and 4). Colorectal cancer patients in advanced stages can be appropriate candidates for palliative care due to the distressful symptoms/conditions accompanied by the disease, such as obstruction, pain, colostomy, odor, and social isolation. ${ }^{14,15}$ There is a prominent non-profit organization, the United Ostomy Associations of America, that supports, empowers, educates, and advocates for 
improving the quality of care and life for patients with a colostomy in the USA. ${ }^{15}$ Evidence indicates that this society is very helpful for alleviating emotional pain related to colostomy. ${ }^{15}$ The possibility that this society can act as an alternative to palliative care during the course of colorectal cancer, particularly when it is not close to death, needs more investigations.

Palliative care was negatively associated with both hospital bed size and location in colorectal cancer. These associations have been reported in many previous studies, ${ }^{18,21-23}$ which indicate that the focus of palliative care promotion has been on large urban-teaching hospitals. Emerging evidence has demonstrated that a dedicated part-time palliative care team can still reduce hospital charges and increase referrals to home hospice in rural hospitals. ${ }^{21}$ Certain interventions should be implemented to increase palliative care access in small and rural hospitals in the USA.

Our study, for the first time, provides insight into details of palliative care, and life-sustaining/local procedures among overall colorectal cancer patients in US hospitals. The utilization of palliative care was $6.9 \%$ while almost $30 \%$ of our patients were metastatic and $46 \%$ had a moderate to severe loss of function. Patients hospitalized in smaller and rural/nonteaching hospitals received less palliative care, which might be partly due to the unavailability of these services in those hospitals. ${ }^{8}$ Minorities did not receive less palliative care in colorectal cancer that contrasts with some other conditions. ${ }^{18}$ Palliative care reduced hospital charges even after controlling for procedures and remained an independent factor for predicting hospital charges. Although palliative care has increased over time, it still seems underutilized in colorectal cancer. Therefore, palliative care should be promoted in all races in colorectal cancer, particularly in metastatic, advanced stages, and small/rural hospitals.

Our study has limitations. We used codes to investigate palliative care and procedures. Errors during the coding process have been reported previously. ${ }^{24}$ However, these errors can have minimal impact on the interpretation of our results due to our large sample size. We could not determine tumor stages since the NIS dataset does not contain such information. The NIS dataset provides deidentified data and readmitted cases are considered new admissions. Therefore, the NIS data are interpretable for each hospitalization, not for a given patient throughout the course of the disease. Almost $12 \%$ of our patients had another diagnosis/comorbidity. The percentage increased from $9.6 \%$ in 2008 to $13.9 \%$ in 2017 , indicating these are mainly chronic illnesses in older people since chronic illnesses have been on the rise in the USA in recent decades. ${ }^{25}$ However, there is still a possibility that patients with cured colorectal cancer have been admitted for some other reasons (eg, hip fracture) with no need for palliative care. The large numbers of our patients minimize the possible effect of this limitation in terms of the main findings.

In conclusion, this study, using ten-year data from the NIS database, shed light on palliative care usage and lifesustaining and local procedures in overall colorectal cancer patients in US hospitals. Palliative care has been increasingly offered to patients with colorectal cancer in inpatient care settings, but it still is underutilized. Palliative care usage was inversely associated with life-sustaining and local procedures. Further studies are warranted to clarify the utilization of these services in subgroups of cancer patients in advanced stages.

\section{Acknowledgment}

This study received no fund from any organizations.

\section{Disclosure}

The authors report no conflicts of interest in this work.

\section{References}

1. Cardona-Morrell M, Kim J, Turner RM, et al. Non-beneficial treatments in hospital at the end of life: a systematic review on extent of the problem. Int $J$ Qual Health Care. 2016;28(4):456-469. doi:10.1093/intqhe/mzw060

2. Barkley R, Soobader MJ, Wang J, Blau S, Page RD. Reducing cancer costs through symptom management and triage pathways. $J$ Oncol Pract. 2019;15(2):e91-e97. doi:10.1200/JOP.18.00082

3. Chan AS, Rout A, D'Adamo CR, Lev I, Yu A, Miller K. Palliative referrals in advanced cancer patients: utilizing the supportive and palliative care indicators tool and Rothman index. Am J Hosp Palliat Care. 2021;10499091211017873. doi:10.1177/10499091211017873

4. Zullig LL, Carpenter WR, Provenzale DT, et al. The association of race with timeliness of care and survival among Veterans Affairs health care system patients with late-stage non-small cell lung cancer. Cancer Manag Res. 2013;5:157-163. doi:10.2147/CMAR. S46688

5. Kalantar-Zadeh K, Wightman A, Liao S. Ensuring choice for people with kidney failure - dialysis, supportive care, and hope. $N$ Engl $J$ Med. 2020;383(2):99. doi:10.1056/NEJMp2001794

6. Hwang J, Shen J, Kim SJ, et al. Ten-year trends of utilization of palliative care services and life-sustaining treatments and hospital costs associated with patients with terminally ill lung cancer in the United States from 2005 to 2014. Am J Hosp Palliat Care. 2019;36 (12):1105-1113. doi:10.1177/1049909119852082

7. Liu X, Shen JJ, Kim P, et al. Trends in the utilization of life-sustaining procedures and palliative care consultation among dying patients with advanced chronic pancreas illnesses in US hospitals: 2005 to 2014. $J$ Palliat Care. 2019;34(4):232-240. doi:10.1177/0825859719827313 
8. Mulvey CL, Smith TJ, Gourin CG. Use of inpatient palliative care services in patients with metastatic incurable head and neck cancer. Head Neck. 2016;38(3):355-363. doi:10.1002/hed.23895

9. Subramoney K, Orman E, Johnson AW, Kara A. The impact of obesity in end of life care in patients with end stage liver disease: an observational study. Am J Hosp Palliat Care. 2020;38:1049909120978768.

10. Zhang B, Wright AA, Huskamp HA, et al. Health care costs in the last week of life: associations with end-of-life conversations. Arch Intern Med. 2009;169(5):480-488. doi:10.1001/archinternmed.20 08.587

11. Chuang MH, Lee FN, Shiau YT, et al. Physician palliative education associated with high use of hospice care service. Am J Hosp Palliat Care. 2021;10499091211014160. doi:10.1177/10499091211014160

12. Rubens M, Ramamoorthy V, Saxena A, et al. Palliative care consultation trends among hospitalized patients with advanced cancer in the United States, 2005 to 2014. Am J Hosp Palliat Care. 2019;36 (4):294-301. doi:10.1177/1049909118809975

13. White A, Joseph D, Rim SH, Johnson CJ, Coleman MP, Allemani C. Colon cancer survival in the United States by race and stage (2001-2009): findings from the CONCORD-2 study. Cancer. 2017;123(Suppl 24):5014-5036. doi:10.1002/cncr.31076

14. Berti-Hearn L, Elliott B. Colostomy care: a guide for home care clinicians. Home Healthc Now. 2019;37(2):68-78. doi:10.1097/ NHH.0000000000000735

15. Miller LR. Ostomy care during hospital stay for ostomy surgery and the United Ostomy Associations of America patient bill of rights: a cross-sectional study. J Wound Ostomy Continence Nurs. 2020;47 (6):589-593. doi:10.1097/WON.0000000000000709

16. Hartman M, Martin AB, Benson J, et al.; National Health Expenditure Accounts Team. National health spending in 2011: overall growth remains low, but some payers and services show signs of acceleration. Health Aff (Millwood). 2013;32(1):87-99. doi:10.1377/ hlthaff.2012.1206
17. Hartman M, Martin AB, Benson J, et al. National Health Expenditure Accounts Team. National health care spending in 2018: growth driven by accelerations in medicare and private insurance spending. Health Aff (Millwood). 2020;39(1):8-17. doi:10.1377/hlthaff.20 19.01451

18. Lee YJ, Yoo JW, Hua L, Kim PC, Kim SJ, Shen JJ. Ten-year trends of palliative care utilization associated with multiple sclerosis patients in the United States from 2005 to 2014. J Clin Neurosci. 2018;58:13-19. doi:10.1016/j.jocn.2018.10.082

19. Cromwell CA, Edwards B, March AL. Improving access to palliative care at a VHA hospital. Am J Hosp Palliat Care. 2021;38 (4):320-325. doi:10.1177/1049909120963079

20. Milki A, Mann AK, Gardner A, Kapp DS, English D, Chan JK. Trends in the utilization of palliative care in patients with gynecologic cancer who subsequently died during hospitalization. Am J Hosp Palliat Care. 2021;38(2):138-146. doi:10.1177/1049909120935038

21. Bakitas MA, Elk R, Astin M, et al. Systematic review of palliative care in the rural setting. Cancer Control. 2015;22(4):450-464. doi:10.1177/107327481502200411

22. Berkowitz CM, Wolf SP, Troy J, Kamal AH. Characteristics of advance care planning in patients with cancer referred to palliative care. JCO Oncol Pract. 2021;17(2):e94-e100.

23. Singh T, Peters SR, Tirschwell DL, Creutzfeldt CJ. Palliative care for hospitalized patients with stroke: results from the 2010 to 2012 national inpatient sample. Stroke. 2017;48(9):2534-2540. doi:10.1161/STROKEAHA.117.016893

24. Feder SL, Redeker NS, Jeon S, et al. Validation of the ICD-9 diagnostic code for palliative care in patients hospitalized with heart failure within the veterans health administration. Am J Hosp Palliat Care. 2018;35(7):959-965. doi:10.1177/1049909117747519

25. Brown CC, Adams CE, George KE, et al. Associations between comorbidities and severe maternal morbidity. Obstet Gynecol. 2020;136(5):892-901. doi:10.1097/AOG.0000000000004057
Cancer Management and Research

\section{Publish your work in this journal}

Cancer Management and Research is an international, peer-reviewed open access journal focusing on cancer research and the optimal use of preventative and integrated treatment interventions to achieve improved outcomes, enhanced survival and quality of life for the cancer patient.
The manuscript management system is completely online and includes a very quick and fair peer-review system, which is all easy to use. Visit http://www.dovepress.com/testimonials.php to read real quotes from published authors. 